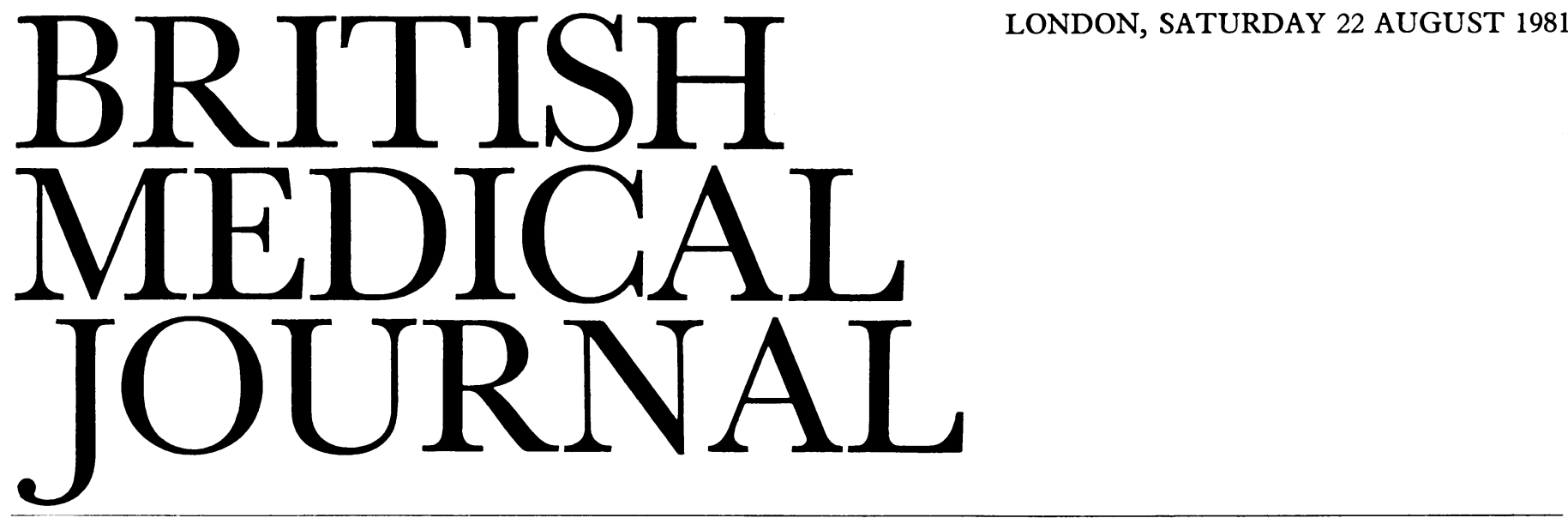

\title{
The new psychiatry
}

The association of depression with disturbance of the hypothalamic-pituitary-adrenal axis has been recognised for a generation, but the precise nature and meaning of these disturbances remain controversial. Diurnal variation of activity, responses to a wide variety of stimuli, and problems of measurement all make standardisation and replication of research findings difficult. ${ }^{12}$ Clinical research is further complicated by the uncertainties of classification of depressive disorders. ${ }^{3}$

Nevertheless, substantial and reproducible neuroendocrine abnormalities have been reported in studies of patients with endogenous depression: these include variations in cortisol secretion; the suppression of cortisol secretion after dexamethasone; the response of luteinising and growth hormones to provocative stimuli; and the response of thyroid-stimulating hormone to thyrotrophin-releasing hormone. ${ }^{4}$

Major depressive disorders have been found to be associated with hypersecretion of cortisol and failure to suppress cortisol secretion after dexamethasone administration. A mean plasma cortisol concentration above $193 \mathrm{nmol} /(7 \mu \mathrm{g} / 00 \mathrm{ml})$ occurs in about half of the patients who meet the research diagnostic criteria for major endogenous depressive disorder. ${ }^{5}$ Moreover, in the more easily undertaken dexamethasone suppression test the serum concentration of cortisol falls below $83 \mathrm{nmol} 1$ (3 $\mu \mathrm{g} 100 \mathrm{ml}$ ) for 24 hours after administration of $2 \mathrm{mg}$ of dexamethasone. ${ }^{6}$ Why this should occur in only half of the patients is not known.

The response of thyroid-stimulating hormone to thyrotrophin-releasing hormone has been less well studied, but evidence is now emerging that patients with unipolar endogenous depression show a decreased or absent response while those with bipolar (manic-depressive) depression show an augmented response to thyrotrophin-releasing hormone. The clinical phenomena of depression are virtually indistinguishable between these two groups. In the manic phase of bipolar illness the response to thyrotrophin-releasing hormone switches from augmented to blunted.

A further piece of the jigsaw is provided by the observation that low pretreatment serum concentrations of 3-methoxy-4hydroxyphenylglycol is associated with a favourable response to imipramine and a failure to respond to amitriptyline. Conversely, those patients with high concentrations of 3 methoxy-4-hydroxyphenylglycol appear to respond well to amitriptyline but not to imipramine. ${ }^{8}$

When these observations are pulled together a scheme can be produced for the laboratory investigation of depressed patients. Without reopening in full the vexed question of the continuum or bimodal model for depressive disorder, this procedure begins by separating patients with primary or endogenous depression into unipolar and bipolar groups and recognising that these are different disorders from those affective disturbances associated with adversity, personal vulnerability, or physical illness.

Next, when a presumptive clinical diagnosis is made of primary depression or when the diagnosis is seriously in doubt the patient should undergo a dexamethasone suppression test and a thyroid-stimulating hormone test would be undertaken. In around half of the cases the dexamethasone suppression test and just over half of the thyroid-stimulating hormone test results would be abnormal; in only $16 \%$ of patients with primary depression would both results be normal. If either test was positive in a doubtful case this should be regarded as strong confirmation of the diagnosis of primary depression, while a negative result should encourage a return to the clinical history and the findings on physical examination. A history of manic disorder or an augmented response in the thyroidstimulating hormone test indicates bipolar disorder likely to respond to lithium carbonate.

Treatment, too, can be guided by biochemical studies. Measurement of 3-methoxy-4-hydroxyphenylglycol in patients with unipolar depression would produce a group with high concentrations who would be expected to respond to amitriptyline and a group with low concentrations who should be treated with imipramine. An abnormal response to the dexamethasone suppression test might also predict those patients requiring adrenaline-augmenting tricyclic antidepressant. Those patients with a normal dexamethasone suppression test and normal concentrations of 3-methoxy-4-hydroxyphenylglycol but an abnormal thyroid-stimulating hormone test might well be the group who require unusual drug combinations, including monoamine-oxidase inhibitors or electric convulsion therapy. Patients who showed a persistent suppression of cortisol response to the dexamethasone suppression test after treatment should probably be maintained on long-term antidepressant therapy. ${ }^{8}$

This persuasive outline of the new psychiatry is derived from recent experimental findings but is not sufficiently established to justify the routine use of these intrusive and expensive tests. In cases of diagnostic or therapeutic difficulty, however, the laboratory data may provide valuable additional information. The use of these tests should now be extended in carefully documented and controlled research studies. Perhaps we can then accumulate sufficient data and experience to establish a reliable routine for investigating depression. Not only would this improve patient care by ensuring a better match between 
drug treatment and the patient's illness but it would also render such treatment more acceptable to those patients and doctors who are more impressed by the results of objective diagnostic tests than by psychiatric advice alone. Finally, the improvement in nosology should have positive effects on research.

\footnotetext{
${ }^{1}$ Ettigi PG, Brown GM. Psychoneuroendocrinology of affective disorder: an overview. Am $\mathcal{F}$ Psychiatry 1977;134:493-501.

2 Sachar EJ, Asnis G, Halbreich U, Nathan RS, Halpern F. Recent studies in the neuroendocrinology of major depressive disorders. Advances in psychoneuroendocrinology. Psychiatric Clinics of North America 1980;3:313-26.

${ }^{3}$ Kendell RE. The role of diagnosis in psychiatry. Oxford: Blackwell Scientific Publications, 1975.

4 Carroll BS. Neuroendocrine dysfunction in psychiatric disorders. In: Lipton MA, DiMascio A, Killam KF, eds. Psychopharmacology. $A$ generation of progress. New York: Raven Press, 1978:487-97.

5 Spitzer RL, Endicott J, Robins E. Research diagnostic criteria. 3rd ed. New York: New York State Division of Mental Health, Biometrics Research, 1977.

6 Gold MS, Pottash ALC, Extein I, Sweeney DR. Diagnosis of depression in the 1980s. FAMA $1981 ; \mathbf{2 4 5}: 1562-4$.

7 Gold MS, Pottash ALC, Ryan N, Sweeney DR, Davies RK, Martin DM. TRH-induced TSH response in unipolar, bipolar and secondary depressions: possible utility in clinical assessment and differential diagnosis. Psychoneuroendocrinology 1980;5:147-55.

${ }^{8}$ Goldberg IK. Dexamethasone suppression test as indicator of safe with-
} drawal of antidepressant therapy. Lancet 1980;i:376.

\section{Primary biliary cirrhosis}

For all its rarity, primary biliary cirrhosis fascinates physicians because of its enigmatic origin, curious clinical picture, and bizarre hepatic lesion. Recent interest has centred on community studies of the disease and its serological marker the antimitochondrial antibody, and on the possibility of treatment by D-penicillamine. Participants at a recent workshop arranged by D R Triger at Sheffield spent much of the time discussing these two aspects.

Last year Triger $^{1}$ reported that nearly $90^{\circ}{ }_{0}$ of his patients came from an area of Sheffield that had only $40 \%$ of the population and was supplied by one particular reservoir. Moreover, the disease was commoner than expected, with an incidence of 5.8 per million and a point prevalence (number of cases in the community at a particular date) of 59 per million. Other speakers gave figures of 8.4 and 40 for Tayside, 6.9 and 50 for Southampton, 12.7 and 83 for the Glasgow area, and 6.0 and 47 for Newcastle upon Tyne. No one so far had found any evidence of clustering to match the Sheffield experience, and Triger had been unable to implicate an environmental factor in the water supply.

One of the difficulties in investigating primary biliary cirrhosis is the variety of pathological changes in the liver. Professor Peter Scheuer characterised the four stages of damage ${ }^{2}$ -florid bile-duct lesions, ductular proliferation, scarring (septal fibrosis and bridging), and cirrhosis-but emphasised that they were uneven and evolved at different speeds in different parts of the liver. Staging, he said, should be based on the most severe lesion present. Antimitochondrial antibodies are present in more than $95 \%$ of patients, ${ }^{3}$ but several speakers pointed out the need for standardisation of assay methods and for agreement on what constitutes a positive titre. This is particularly important in community surveys, where a few apparently healthy people are found to be positive for antimitochondrial antibody; a proportion of these can be shown to have the histological changes of primary biliary cirrhosis if subjected to liver biopsy. ${ }^{4}$

The importance of a firm diagnosis and proper staging is underlined by the possibility of treatment. Until recently little could be done except for relieving the intense itching by cholestyramine, and trying to prevent the demineralisation of bones from malabsorption of calcium and vitamin $\mathrm{D}$ resulting from chronic obstructive jaundice. Treatment with D-penicillamine was originally considered because of its chelating properties $^{5}$ : in the late stages of the disease accumulation of copper due to cholestasis reaches levels comparable with those seen in Wilson's disease. The drug may also have immunological and antifibrotic effects. At the Sheffield meeting Epstein reported guidelines for the treatment of primary biliary cirrhosis based on experience in the Royal Free Hospital trial. ${ }^{6}$ A starting dose of $150 \mathrm{mg}$ D-penicillamine daily is built up by monthly increments to $600 \mathrm{mg}$ while a careful watch is kept for side effects, such as rashes, neutropenia, and proteinuria. Little benefit is discernible up to 18 months, but thereafter survival is improved in patients with late-stage disease. While some improvement in liver function tests and histological damage appears when patients so treated are compared with controls, these procedures have not, unfortunately, proved of help in monitoring responses in individual patients. Symptomless patients and those with stage I and stage II disease need not be treated, as the prognosis is generally good in these groups; but ideally they should have a liver biopsy performed every year or so to detect any deterioration.

1 Triger DR. Primary biliary cirrhosis: an epidemiological study. Br Med $\mathcal{F}$ $1980 ; 281: 772-5$

2 MacSween RN. Primary biliary cirrhosis. In : MacSween RN, Anthony A, Scheuer PJ, eds. Pathology of the liver. Edinburgh: Churchill, 1979 306-14.

${ }^{3}$ Munoz LE, Thomas HC, Scheuer PJ, Doniach D, Sherlock S. Is mitochondrial antibody diagnostic of primary biliary cirrhosis ? Gut 1981 ;22: 136-40.

' James O, Macklon AF, Watson AJ. Primary biliary cirrhosis-a revised clinical spectrum. Lancet $1981 ; \mathrm{i}: 1278-81$.

5 Jain S, Scheuer PJ, Samourian S, McGee JO'D, Sherlock S. A controlled trial of D-penicillamine therapy in primary biliary cirrhosis. Lance $1977 ; \mathrm{i}: 831-4$.

${ }^{6}$ Epstein O, Jain S, Lee RG, et al. D-penicillamine treatment improves survival in primary biliary cirrhosis. Lancet $1981 ; 1: 1275-7$.

\section{Haemolytic disease of the newborn due to antibodies other than rhesus anti-D}

Perinatal mortality from haemolytic disease has fallen dramatically in the past 10 years. Stillbirths and neonatal deaths from this condition are almost always associated with alloimmunisation of the mother to the rhesus antigen $\mathrm{D}$, and the decline has been due to two main factors. One is the smaller size of families, stillbirth from haemolytic disease tending to occur in later pregnancies. The other, and more important, is that since about 1968 alloimmunisation to $\mathrm{D}$ has been largely prevented by giving anti-D immunoglobulin to the $\mathrm{Rh}$ negative mother immediately after delivery of an Rh-positive baby. Such prophylactic treatment does not, however, prevent alloimmunisation by antigens other than $\mathrm{D}$, so that cases of haemolytic disease of the newborn from antibodies other than rhesus anti-D are becoming proportionally more important.

A survey by Giblett ${ }^{1}$ carried out before prophylactic treatment was introduced found that $7^{\circ} \%$ of 420 cases of haemolytic disease in Washington were caused by antibodies other than anti-D or anti-C + D. Almost all of these were in babies born to $\mathrm{Rh}$-positive mothers, and in about half the antibodies 